\title{
AVOGADRO'S LAW DEDUCED FROM THE FUNDAMENTAL PRINCIPLE OF THE MECHANICAL THEORY OF GASES.
}

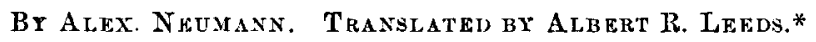

AVOGADRo's LAW, that equal volumes of different gases at the same pressure and temperature contain an equal number of molecules, is certainly prized by the majority of scientific chemists as the surest foundation of those molecular and atomic weights, which permit the chemical constitution and reactions to be expressed in the simplest manner. It might, therefore, not be without value to prove that we are necessarily led from an altogether different point of departure, and upon an altogether different road, to the same law.

The pressure of gases is the sum of all the impacts, which the molecules in consequence of their progressive motion exert upon bounding surfaces. The pressure upon the surface unit will therefore depend-1. Upon the mass of the individual molecule. 2. Upon its velocity. 3. Upon the gas volume. 4. Upon the number of molecules present. In one case let the mass of the molecule be $m$ and its velocity $c$, in another its mass $M$ and its velocity C. Since the magnitude of the impact stands in direct relation to the mass and velocity, it is proportional to the magnitude of both or the momentum, i.e., $\frac{m c}{\mathrm{M} \mathrm{C}}$. Again, the number of impacts made by a molecule in equal times is proportional to its velocity, i.e., $=\frac{c}{\mathrm{c}}$. Therefore, the pressures relate themselves in regard to masses and velocities as -

$$
\frac{p}{\mathrm{P}}=\frac{m c^{2}}{\mathrm{MC}^{2}}=\frac{\frac{m c^{2}}{2}}{\mathrm{MC}^{2}}, . \quad \text {. . . . . . }
$$

2

If we imagine $n$ molecules at equal distances and in a state of rest in a space of the cubic capacity $v$, then there are in contact with the cubic unit $n_{1}={ }_{v}^{n}$ molecules; with a line equal to the unit of length $l=\sqrt{\frac{n}{v}}$, and with the square unit $f=(\sqrt[3]{\sqrt[n]{v}})^{2}$ molecules. If now, the number of molecules remaining the same, the volume

* Ber. der Deutsch. Chem, Gesellsch. zu Berlin, page 690, 1869.

Vol. LIX.-ThIRD SkrIHs.-No. 4.-A PrIL, 1870. 
$v$ is changed to volume $\mathrm{v}$, then there will be in contact with the cubic unit, $n_{2}={ }_{\mathrm{v}}^{n}$ molecules, with the unit of length $\mathrm{L}=\sqrt[3]{\mathrm{v}^{2}}$, and with the square unit $\mathrm{F}=(\sqrt[3]{\sqrt{\mathrm{v}}})^{2}$. Now, in a state of motion, the pressure must be proportional to the number of molecules imping. ing upon the square unit in equal times, i.e. proportional both to the number of molecules in contact with it at a certain instant, and also to the number of molecules along the unit of length, because the surface unit will be impinged upon so many times more frequently as the distance of the molecules one from another is less or-

$$
\mathrm{P}=\frac{f l}{\mathrm{FL}}=\frac{\left(\sqrt[3]{\frac{n}{n}}\right)^{2} \sqrt[3]{\frac{n}{v}}}{\left(\sqrt[3]{\frac{n}{\mathrm{v}}}\right)^{2} \sqrt[3]{\frac{n}{\mathrm{v}}}}=\frac{\frac{n}{v}}{\frac{n}{\mathrm{~V}}}=\frac{\mathrm{v}}{v}
$$

From equation (2) follows also $\frac{p}{\mathrm{P}}=\frac{n}{v} \frac{\mathrm{v}}{n}=\frac{n_{1}}{n_{2}}$. Since this ratio $\frac{n_{1}}{n_{2}}$ of the molecules contained in the volume unit is equal to the ratio $\frac{n}{\mathrm{~N}}$ of the number of molecules contained in equal volumes $v=\mathrm{v}$, so for equal volumes is-

$$
\frac{p}{\mathrm{P}}=\frac{n}{\mathrm{~N}}, \quad \cdot \quad \cdot \quad \cdot \quad \cdot \quad \cdot \quad \cdot \quad .
$$

If the volumes as well as the number of molccules are different, we shall obtain from equations (2) and (3)-

$$
\frac{p}{\mathrm{P}}=\frac{n \mathrm{~V}}{\mathrm{~N} v}, . \quad . \quad . \quad . \quad . \quad \text {. . . . }
$$

Moreover, from equations (4) and (1) we shall bave-

$$
\frac{p}{\mathrm{P}}=\frac{n \nabla \frac{m c^{2}}{2}}{\mathrm{~N} v \frac{\mathrm{M} c^{2^{2}}}{2}}
$$

According to the experimental law of Mariotte and Gay Lussac, we have for the same gas the volume remaining constant-

$$
\frac{p_{1}}{\mathrm{P}_{2}}=\frac{n_{1}}{n_{2}} \frac{273+t_{1}}{273+t_{2}}=\frac{n_{1} \mathrm{~T}_{1}}{n_{2} \mathrm{~T}_{2}}, \quad \text {. . . . . }
$$

in which the ratio of the numbers $n_{1}$ and $n_{2}$ of the molecules contained in equal volumes, expresses at the same time the density; 
$\mathrm{T}_{1}$ and $\mathrm{T}_{2}$ indicate the so-called absolute temperatures-numbered on from $-273^{\circ} \mathrm{C}$.

If we denote, however, for equal volumes of the same gas the velocities of the molecules, corresponding to the absolute tempera. tures $\mathrm{T}_{1}$ and $\mathrm{T}_{2}$, equation 5 becomes-

$$
\frac{p_{1}}{p_{2}}=\frac{n_{1} \frac{m c_{1}^{2}}{2}}{n_{2} \frac{m c_{2}^{2}}{2}}, \text {. . . . . . . . . . . . . }
$$

From equations (6) and (7) we have-

$$
\frac{\frac{m c_{1}^{2}}{2}}{\frac{m c_{2}^{2}}{2}}=\frac{\mathrm{T}^{1}}{\mathrm{~T}_{2}}
$$

When different gases, which do not react together of the same temperature are mingled, at a temperature, for example, $\mathrm{T}_{2}$, this temperature remains unaltered, being independent of the relative quantities of the mixed gases and of their volumes. Therefore, at the same temperature, the vis viva of the moving molecules must be equally great in difierent gases, i. e.-

$$
\frac{m c_{2}^{2}}{2}=\frac{\mathrm{M} \mathrm{C}_{2}^{2}}{2^{2}}, \text {. . . . . . . . }
$$

From equations (8) and (9) we have-

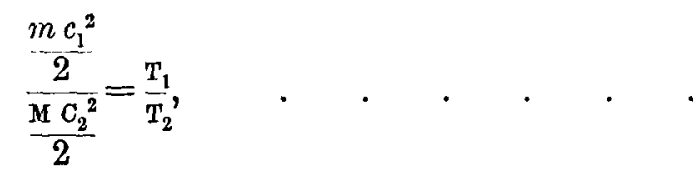

If, for the ratio of the vis vivas in equation (5) we substitute the ratio of the absolute temperatures according to equation (10) we shall have-

$$
\frac{p}{\mathrm{P}}=\frac{n v \mathrm{~T}_{1}}{N v \mathrm{~T}_{2}}
$$

Finally, we make in equation (11)-

$$
p=\mathrm{P}, \mathrm{T}_{1}=\mathrm{T}_{2}, v=\mathrm{V}
$$

we shall have-

$$
n=\mathrm{N}, \quad \cdot \quad \cdot \quad \cdot \quad \cdot \quad .
$$

i.e., at the same pressure and at the same temperature, equal volumes of different gases contain the same number of molecules. 